\title{
The Cost of Bottling It Up: Emotion Suppression as a Mediator in the Relationship Between Anger and Depression Among Men with Prostate Cancer
}

This article was published in the following Dove Press journal: Cancer Management and Research

\author{
Simon M Rice $\mathbb{D}^{1,2}$ \\ David Kealy (D) ${ }^{3}$ \\ John S Ogrodniczuk ${ }^{3}$ \\ Zac E Seidler ${ }^{1,2}$ \\ Linda Denehy ${ }^{4,5}$ \\ John L Oliffe (iD ${ }^{6,7}$ \\ 'Orygen, Melbourne, VIC, Australia; \\ ${ }^{2}$ Centre for Youth Mental Health, The \\ University of Melbourne, Melbourne, \\ VIC, Australia; ${ }^{3}$ Department of \\ Psychiatry, University of British \\ Columbia, Vancouver, BC, Canada; \\ ${ }^{4}$ School of Health Sciences, The \\ University of Melbourne, Melbourne, \\ VIC, Australia; ${ }^{5}$ Department of Allied \\ Health, Peter MacCallum Cancer Centre, \\ Melbourne, VIC, Australia; ${ }^{6} \mathrm{School}$ of \\ Nursing, University of British Columbia, \\ Vancouver, BC, Canada; ${ }^{7}$ Department of \\ Nursing, The University of Melbourne, \\ Melbourne, VIC, Australia
}

Background: Prostate cancer is a risk factor for major depressive disorder. Recent psychooncology research suggests a potential role for male-specific mood-related symptoms in this relationship. Gender socialisation experiences may reinforce men's anger and emotion suppression responses in times of distress, and anger and emotion suppression may be implicated in pathways to, and maintenance of depression in prostate cancer.

Patients and Methods: Data were collected online from men with a self-reported diagnosis of prostate cancer ( $\mathrm{N}=100$; mean age 64.8 years). Respondents provided information regarding diagnosis and treatment, in addition to current experience of major depression and male-specific externalising symptoms.

Results: Prostate cancer diagnosis in the last 12 months occurred for $35.4 \%$ of the sample. Elevated major depression symptoms were observed for $49 \%$ of respondents, with $14 \%$ endorsing past 2-week suicide ideation. Parallel mediation analysis (99\% CIs) controlling for prostatectomy and active surveillance indicated men's emotion suppression mediated the relationship between anger and depression symptoms $\left(R^{2}=0.580\right)$. Trichotimised emotion suppression scores with control variables yielded a large multivariate effect $(p<0.001$, partial $\eta^{2}=0.199$ ). Univariate moderate-sized effects were observed for emotion suppression comparisons for symptoms of depressed mood and sleep disturbance, and a large effect observed for guilt-proneness.

Conclusion: Findings highlight the salience of anger in the experience of depression symptoms for men with prostate cancer. The mediating role of emotion suppression, which in turn was strongly linked to men's feelings of guilt, suggests potential assessment and intervention targets. Future work should examine the role of androgen deprivation therapy and other treatments including active surveillance on the relationship between anger and depression in men with prostate cancer. Consideration of interventions focused on emotion processing skills in psycho-oncology settings may help reduce men's reliance on emotion suppression as a strategy for coping with feelings of anger or guilt in the context of prostate cancer.

Keywords: prostate, depression, anger, emotion suppression

Men diagnosed with prostate cancer have a heightened risk for major depressive disorder, ${ }^{1,2}$ with rates exceeding those seen in the general male population. ${ }^{3,4}$ This finding has been linked to the unmet psychosocial needs of this population following diagnosis, ${ }^{5}$ in addition to prostate cancer-related symptoms, ${ }^{6}$ and treatmentinduced side effects including sexual dysfunction and urinary incontinence. ${ }^{7-9}$ Even those men on active surveillance are at high risk for depression ${ }^{10}$ whereby a decline
Correspondence: Simon M Rice

Centre for Youth Mental Health, The

University of Melbourne, Melbourne,

Australia

Tel +61399669100

Email simon.rice@unimelb.edu.au 
in quality of life can be expected in the first 6 months following diagnosis. ${ }^{11}$ Depression among men with prostate cancer is also associated with the loss of masculine identity. ${ }^{12}$ Amid efforts to ensure depression does not by extension go undetected or untreated in men with prostate cancer, ${ }^{4}$ symptom screening is recommended. ${ }^{13}$ Such approaches are essential given men with depression have an increased risk of prostate cancer mortality ${ }^{14}$ and suicide. $^{15}$

A diagnosis of major depression is made on the basis of the nine diagnostic criteria per DSM- $5^{16}$ or ICD- $10 .{ }^{17}$ There is growing concern that these diagnostic criteria may inaccurately reflect men's experiences of mood disturbance, and therefore insufficiently identify men with an underlying mood disorder. ${ }^{18,19}$ Studies support the existence of a phenotypic variant of depression in men, ${ }^{20,21}$ characterised by externalising and atypical symptoms including anger, ${ }^{22}$ substance use, ${ }^{23}$ risk-taking ${ }^{24}$ and emotion suppression. ${ }^{25}$ These symptom domains tend to be socially sanctioned for men as reflecting culturally informed and affirmed dominant Western masculine norms (as opposed to vulnerable emotions of sadness) ${ }^{26}$ It is unclear whether this range of externalising and atypical symptoms reflect problematic coping strategies and a predisposing risk factor for depression in men, or should instead be considered part of the prodromal phase on a unique manifestation of depression in men. There is evidence suggesting that such symptoms may be a unique feature of men's depression, ${ }^{21}$ especially in the context of recent depressogenic negative life events that may precipitate major mood disturbance. ${ }^{27}$ Nonetheless, it is important to recognise that this research area is in a nascent stage and draws debate. ${ }^{28}$

Regardless of the role of externalising symptoms in men's depression, in the context of oncology - and prostate cancer more specifically - the emotion and expression of negative affect (such as anger) has been identified as particularly important. ${ }^{29,30}$ Amid growing literature on the role of anger in other cancers, such as breast cancer, ${ }^{31-34}$ recent calls have been made to further anger-based research in the context of prostate cancer. ${ }^{35}$ Importantly, research has shown an association between morbidity and suppression of negative emotion $^{36-38}$ and a link has been reported between prostate cancer and suppression of anger, ${ }^{39}$ though corroborating evidence at present is sparse. ${ }^{40}$ While psychodynamic theory has long posited that anger turned inwards can precipitate depression, ${ }^{41}$ anger has largely been ignored by other treatment models in the conceptualisation and treatment of emotional disorders such as depression. ${ }^{42}$ This negligence has occurred despite the fact that anger predicts negative consequences, greater symptom severity and worse treatment response for such conditions. ${ }^{43}$

In the context of potential linkages between anger and depression in men with prostate cancer, the present study explored five putative male-specific coping strategies (emotion suppression, drug use, alcohol use, somatisation, risk-taking) as parallel mediators ${ }^{44}$ in the relationship between anger and symptoms of major depressive disorder. Consistent with previous research, ${ }^{45,46}$ significant associations were expected between externalising and atypical symptoms of depression. Based on the findings of others, ${ }^{36,39}$ it was hypothesised that emotion suppression would mediate the relationship between anger and depression, and that alcohol and drug use may also be implicated (as external coping strategies) in this relationship.

\section{Method \\ Design and Patients}

Secondary analysis was undertaken on data previously used for a psychometric study based on a cross-sectional online survey, with the detailed method reported elsewhere. ${ }^{47}$ In brief, recruitment occurred online in 2017 via a prostate cancer help website and social media channels. Participants were recruited as part of a convenience sample of 100 Canadian men with prostate cancer.

\section{Materials}

Participants provided background information including country and province, age, employment status, education level, sexual identity, cultural affiliation, year diagnosed with prostate cancer and year treated, prostate cancer treatment(s), and current treatment. Data for symptoms of major depressive disorder (MDD) were collected via the PHQ-9. ${ }^{48}$ The PHQ-9 items reflect the MDD diagnostic criteria in the DSM-V (American Psychological Association [APA], 2013). Respondents indicated how often ( $0=$ "'not at all", $1=$ "'several days', $2=$ "'more than half the days' and $3=$ "nearly every day") they had experienced any of the listed symptoms over the previous 2 weeks (see Table 1 for items). Total scores reflect symptom severity: mild depression (total scores 5-9), moderate depression (total scores 10-14), moderately severe depression (total scores 15-19) and severe depression (total scores 20-27) with the scale demonstrating internal consistency in the present sample $(\alpha=0.89)$. Previous research has established the reliability and validity of the PHQ- $9 .{ }^{49}$ Male-specific 
Table I Demographic and Outcomes for Total Sample and by Emotion Suppression Grouping

\begin{tabular}{|c|c|c|c|c|c|c|c|}
\hline & & \multirow[t]{2}{*}{ Total $(\mathrm{N}=100)$} & \multicolumn{3}{|c|}{ Emotion Suppression Group } & \multicolumn{2}{|c|}{ Inferential } \\
\hline & & & Low $(n=28)$ & Moderate $(n=42)$ & High $(n=30)$ & Statistic & $p$ \\
\hline \multicolumn{8}{|l|}{ Background variables } \\
\hline Age (years) & $M(S D)$ & $64.81(7.17)$ & $65.96(6.35)$ & $65.74(7.60)$ & $62.43(6,95)$ & $F=2.43$ & 0.094 \\
\hline Current treatment & $(\%, n)$ & $33.0(33)$ & $32.1(9)$ & $31.0(13)$ & $36.7(\mathrm{II})$ & $\chi^{2}=0.27$ & 0.873 \\
\hline Prostatectomy & $(\%, n)$ & $54.0(54)$ & $46.4(13)$ & $69.0(29)$ & $40.0(12)$ & $\chi^{2}=6.84$ & 0.033 \\
\hline Androgen deprivation & $(\%, n)$ & $18(18)$ & $17.9(5)$ & $23.8(10)$ & 10.0 & $\chi^{2}=2.26$ & 0.323 \\
\hline Active surveillance & $(\%, n)$ & $22.0(22)$ & $21.4(6)$ & $22.7(5)$ & $36.7(\mathrm{II})$ & $\chi^{2}=6.19$ & 0.044 \\
\hline Past year diagnosis & $(\%, n)$ & $35.0(35)$ & $21.4(6)$ & $35.7(15)$ & $48.3(14)$ & $\chi^{2}=4.50$ & 0.106 \\
\hline Years since diagnosis & $M(S D)$ & $4.59(5.07)$ & $5.79(5.7 \mathrm{I})$ & $4.83(5.17)$ & $4.59(5.06)$ & $F=2.12$ & 0.127 \\
\hline Comorbidity & $(\%, n)$ & $38.0(38)$ & $35.7(10)$ & $31.0(13)$ & $50.0(15)$ & $\chi^{2}=2.78$ & 0.249 \\
\hline \multicolumn{8}{|l|}{ Clinical variables } \\
\hline PHQ-9 Total & $M(S D)$ & $5.85(5.57)$ & $2.14(2.65)$ & $5.45(5.29)$ & $9.87(5.46)$ & $F=19.29$ & $<0.001$ \\
\hline Suicide ideation & $(\%, n)$ & $14(14)$ & $0(0)$ & $28.6(4)$ & $33.3(10)$ & $\chi^{2}=14.57$ & $<0.001$ \\
\hline Alcohol Use & $M(S D)$ & 2.51 (4.97) & $0.96(3.67)$ & 2.07 (4.49) & $4.57(6.03)$ & $F=4.37$ & 0.015 \\
\hline Anger & $M(S D)$ & $3.53(5.18)$ & $1.32(2.57)$ & 2.48 (3.91) & 7.07 (6.69) & $F=12.88$ & $<0.001$ \\
\hline Emotion Suppression & $M(S D)$ & $8.73(7.73)$ & $0.64(0.87)$ & $7.05(3.47)$ & I8.63 (7.73) & $F=232.79$ & $<0.001$ \\
\hline Drug Use & $M(S D)$ & $1.03(3.24)$ & $0.36(1.06)$ & $1.19(3.84)$ & $1.43(3.64)$ & $F=0.89$ & 0.416 \\
\hline Somatic Symptoms & $M(S D)$ & $5.14(5.35)$ & $1.96(3.70)$ & $5.55(5.44)$ & $7.53(5.24)$ & $F=9.4 I$ & $<0.001$ \\
\hline Risk-Taking & $M(S D)$ & $1.30(2.6 I)$ & $0.36(1.70)$ & $1.05(2.15)$ & $2.53(3.38)$ & $F=5.92$ & 0.004 \\
\hline
\end{tabular}

Note: Bolded text denotes significant at $p<0.05$.

Abbreviations: M, Mean; SD, Standard deviation.

depression symptoms were assessed via the Male Depression Risk Scale (MDRS-22), ${ }^{45,46}$ which comprises 22 items and six subscales assessing anger $(\alpha=0.92)$, alcohol use $(\alpha=0.91)$, drug use $(\alpha=0.91)$, emotion suppression $(\alpha=0.88)$, somatisation $(\alpha=0.67)$, risk-taking $(\alpha=0.59)$. MDRS-22 items refer to the previous four weeks $(0=$ "not at all" to 7="almost always"). The MDRS-22 has been cross-nationally validated, ${ }^{45,46}$ and demonstrates test-retest validity and sensitivity to change. ${ }^{27}$

\section{Procedure}

Ethics review was approved by the University of British Columbia (H12-00573). The survey was launched in January 2017, focussing on the issue of depression in men with prostate cancer. Participation provided an opportunity for respondents to be entered into a $\$ 500$ cash prize draw by completing the survey. The survey was embedded in an online prostate cancer psychosocial resource (www.ifiwere tom.ca) and was available for 3 months through April 2017. The survey landing page provided details about the study including informed consent, confidentiality regards respondent's demographic data and its separate password-protected storage from their survey responses. On completion of the survey, respondents were provided a URL link to the men's depression website www.headsupguys.org

\section{Data Analysis}

Parallel mediation analysis was conducted using the PROCESS macro version 3.2.01 ${ }^{44}$ in SPSS 25.0. The model tested five male-specific symptoms (emotion suppression, drug use, alcohol use, somatisation, risk-taking) as parallel mediators in the relationship between anger (predictor variable) and symptoms of major depressive disorder (dependent variable; PHQ-9 total score). The model included covariates, identified according to subgroup differences for emotion suppression (the potential covariates were; age, active surveillance, comorbidity, current treatment, recency of diagnosis, prostatectomy) and reported $99 \%$ confidence intervals for the bootstrapped estimates conducted with 10,000 resamples. To further explore effects in a sensitivity analysis, emotion suppression scores were trichotimised, with the three groups subsequently used in a between-subjects MANCOVA, with the nine individual symptoms of depression (as assessed by the PHQ-9) examined as dependent variables. As per the mediation analysis, covariates were entered where group differences existed. In order to identify the unique contribution of emotion suppression on individual depression symptoms, the remaining male-specific symptoms were also entered as covariates (eg, anger, drug use, alcohol use, somatisation, risk-taking). Effect sizes were 
reported using the partial eta-squared coefficient, following accepted interpretation of small (partial $\eta^{2}=0.01-0.05$ ), medium (partial $\left.\eta^{2}=0.06-0.13\right)$ and large (partial $\left.\eta^{2} \geq 0.14\right)$ effects. $^{50}$

\section{Results}

The mean age of participants was 64.8 years (range 47-85 years). A total of $35.4 \%$ of the sample reported a prostate cancer diagnosis in the last 12 months. Elevated major depression symptoms (PHQ-9 $\geq 5$ ) were observed for $49 \%$, with $14 \%$ endorsing past 2 -week suicide ideation. Other demographic data are presented in Table 1.

Parallel mediation analysis was conducted including the two control variables with a significant between-group difference (eg, prostatectomy, surveillance). The model accounted for a large portion of variance in depression symptoms $\left(R^{2}=0.580\right)$. As predicted, emotion suppression was a significant mediator (indirect effect $=0.327$, $p<0.001)$ in the relationship between anger and depression symptoms. For a one-unit increase in men's anger, depression increased by 0.327 units through the mediating effect of emotion suppression. No other male-specific coping strategies were significant mediators and the covariates were not significant in the model (see Table 2). When examined as unique predictors of depression (excluding covariates,) anger accounted for $34.6 \%$ of variance in depression symptoms with emotion suppression accounting for an additional $15.4 \%$ beyond that accounted for by anger.

MANCOVA with the individual depression symptoms indicated a significant, large multivariate effect of emotion suppression $\lambda=0.660, F(18,164)=2.101, p<0.001$, partial $\eta^{2}=0.187$, with anger a significant multivariate covariate $\left(p=0.039\right.$, partial $\left.\eta^{2}=0.187\right)$. Figure 1 depicts this multivariate effect whereby symptoms of depression varied according to severity of emotion suppression. At the univariate (eg, individual symptom) level, significant effects were seen for depressed mood $\left(p<0.001\right.$ partial $\eta^{2}=0.155$; anger as covariate $p=0.023$, partial $\eta^{2}=0.064$ ), sleep disturbance $\left(p=0.033\right.$, partial $\eta^{2}=0.073$; anger as covariate $p=0.022$, partial $\left.\eta^{2}=0.057\right)$, and guilt proneness $(p<0.001$, partial $\eta^{2}=0.223$; anger as covariate $p=0.006$, partial $\eta^{2}=0.081$ ). Bonferroni corrected post hoc analysis indicated five significant sub-group comparisons at the symptom level for these three symptom domains (see Figure 1).

\section{Discussion}

The principal findings of the present study revealed a significant association between anger and depressive
Table 2 Coefficients for Direct and Indirect Effects of Anger Predicting Depression Through Male-Specific Symptoms as Potential Mediators ( $99 \% \mathrm{Cls}$ )

\begin{tabular}{|c|c|c|c|c|}
\hline & B & SE & $t$ & $P$ \\
\hline DV: Emotion Suppression & & & & \\
\hline Anger & 0.807 & 0.129 & 6.246 & 0.004 \\
\hline Active surveillance $^{\#}$ & 1.889 & 1.929 & 0.979 & 0.330 \\
\hline Prostatectomy $\#$ & 0.525 & 1.599 & 0.743 & 0.743 \\
\hline Model summary & \multicolumn{4}{|c|}{$F(3,96)=14.798, R^{2}=0.316, p<0.001$} \\
\hline \multicolumn{5}{|l|}{ DV: Depression } \\
\hline Anger & 0.285 & 0.111 & 2.580 & 0.012 \\
\hline Emotion suppression & 0.327 & 0.063 & 5.176 & $<0.001$ \\
\hline Drug use & 0.223 & 0.122 & 1.838 & 0.070 \\
\hline Alcohol use & -0.157 & 0.082 & -1.919 & 0.058 \\
\hline Somatisation & 0.146 & 0.082 & 1.784 & 0.079 \\
\hline Risk-taking & 0.083 & 0.203 & 0.411 & 0.682 \\
\hline Active surveillance $^{\#}$ & -0.761 & 1.186 & -0.642 & 0.523 \\
\hline Prostatectomy $\#$ & -1.743 & 0.973 & -1.792 & 0.076 \\
\hline Model summary & \multicolumn{4}{|c|}{$F(8,9 \mid)=15.740, R^{2}=0.581, p<0.001$} \\
\hline & \multicolumn{4}{|c|}{ Bootstrap estimate } \\
\hline Indirect effects & Effect & SE & Lower $\mathrm{Cl}$ & Upper Cl \\
\hline DV: Depression & & & & \\
\hline $\begin{array}{l}\text { Anger through Emotion } \\
\text { suppression }\end{array}$ & 0.241 & 0.078 & 0.058 & 0.470 \\
\hline Anger though Drug use & 0.007 & 0.023 & -0.051 & 0.094 \\
\hline Anger through Alcohol use & -0.036 & 0.033 & -0.165 & 0.012 \\
\hline $\begin{array}{l}\text { Anger through Somatic } \\
\text { symptoms }\end{array}$ & 0.060 & 0.044 & -0.036 & 0.206 \\
\hline Anger through Risk-taking & 0.027 & 0.087 & -0.181 & 0.315 \\
\hline
\end{tabular}

Notes: ${ }^{*}$ covariate, $\mathrm{N}=100, \mathrm{Cl}=99 \%$ confidence interval ( 10,000 resamples), boldface text indicates statistically significant values at $p<0.01$.

Abbreviation: DV, Dependent variable.

symptoms among men with prostate cancer, and that emotion suppression is a reliable mediator of this relationship. Emotion suppression accounted for an additional 15\% of depression variance beyond anger, with the two variables accounting for large $(50 \%)$ proportion of variance in depression for this sample. This was a robust finding given that four other mediators were examined simultaneously, in addition to controlling for the effects of covariates. While it is not clear from the present data whether participants were specifically suppressing anger or other negative emotions (eg, sadness, guilt), the present findings do align with the notion that anger turned inward may precipitate depression. ${ }^{41}$ Further research should seek to explore (and indeed differentiate) suppression of negative emotion versus anger more specifically. Nonetheless, present findings suggest that the potential importance of considering constricted or internalised anger in the 

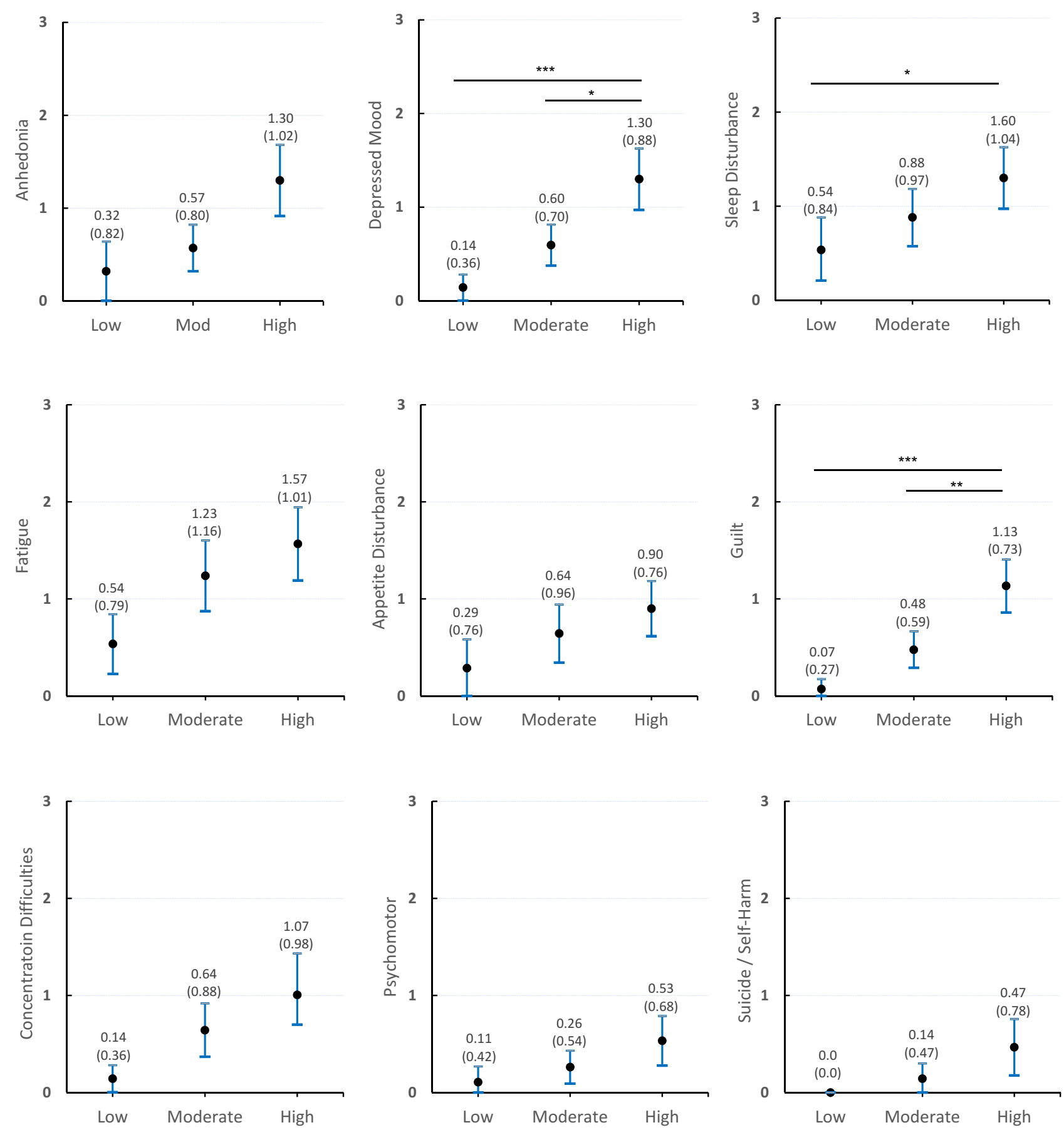

Figure I Mean depression symptoms (with 95\% Cls) by emotion suppression group including Bonferroni corrected post hoc tests.

Notes: *** $p<0.001$, ** $p<0.01$, *p<0.05; Y-axis represents PHQ-9 response scale (over previous 2-weeks); $0=$ "Not at all”, I="Several days", $2=$ "More than half the days", $3=$ "Almost every day". Several "apparent" group differences did not survive Bonferroni correction.

psychosocial context of prostate cancer. The findings additionally point to the critical role of emotion suppression as a mechanism through which anger influences the development of depressive symptoms. Recent survey research with a sample of 4000 adult men from the US, UK, Canada and Australia reported that a large proportion of respondents regretted sharing their emotions with others, conceding that they would be unlikely to do so again as a consequence of that decisional regret. ${ }^{51}$ While that sample did not specifically include men with prostate cancer, this outcome is concerning in light of the present study which suggests that suppressing (rather than sharing or reappraising strong emotions such as anger) can lead to more severe moodrelated symptoms consistent with depression. 
The multivariate effect was robust across the nine depression symptoms, with corrected post hoc tests revealing significant group differences across three of the symptom domains (depressed mood, sleep disturbance and guilt proneness). While the proportion of respondents endorsing past 2-week suicide or self-harm ideation differed across the groups, there were no differences for the adjusted mean comparisons of suicide or self-harm ideation. Nonetheless, that $0 \%$ of the low emotion suppression group reported recent suicide or self-harm ideation whereas $28.6 \%$ and $33.3 \%$ of the moderate and high groups did, suggests that emotion suppression may be problematic for men with prostate cancer.

While longitudinal designs are needed to corroborate the temporal relationship between anger and depression in prostate cancer, the present findings have a range of practical implications. Prostate cancer can result in a loss of masculine identity for men, ${ }^{12}$ and perceptions of diminished masculine identity can be triggered by a sense of dependence, loss of control and feelings of weakness. ${ }^{52}$ It is possible that anger and emotion suppression may be operating as socially sanctioned emotional responses for men with prostate cancer, in part offering ways to retain (albeit problematically) these gender-related changes, or overcoming a sense of "wounded" masculinity. ${ }^{53}$ Educating men that suppressing distressing emotions, like anger, may increase the likelihood of experiencing major depression and open the door to developing more adaptive responses to distress. Acceptance-based approaches may be useful for men with a tendency to suppress emotions, as early work has shown positive effects for such approaches in reducing avoidance in men with a prostate cancer diagnosis. ${ }^{54}$ Supporting emotion-based skill development in this population is also likely to assist. For example, helping men more adaptively process anger-based emotions could help mitigate aggressive responses to feeling angry that often lead to negative consequences (eg, impaired interpersonal relationships, perceived coercion), ${ }^{55}$ and thereby potentially reduce the burden of depression among men with prostate cancer. Any gender and age-sensitised approaches should be mindful of socialisation processes that may contribute to men's reluctance to making changes. Hence, leveraging and working with (rather than attempting to change) masculine norms may be important in implementing sensitised strength-based approaches for men with prostate cancer, while considering key social determinants and health inequities among sub-groups of men. ${ }^{56}$

In terms of limitations, as the present sample did not provide data on previous mental health diagnoses (including previous depression diagnosis or treatment), it is not possible to determine the rate of relapses versus incident cases of depression. Similarly, related mental disorders such as generalised anxiety and substance dependence were not assessed. While half $(54 \%)$ of the sample reported prostatectomy and the average time elapsed since first prostate diagnosis was 4.6 years, findings for the anger-emotion suppression-depression relationship may be further influenced and/or moderated by factors such as social support, dispositional optimism and response to treatment or sideeffects. It is recommended that future work examine these factors in larger samples, including a specific focus on those men receiving treatment modalities and combinations including androgen deprivation therapy. Further, it is widely known that there is significant overlap between symptoms of anxiety and symptoms of depression; ${ }^{43}$ thus future research should assess for comorbid anxiety symptoms in men with prostate cancer. From a design perspective, the present data were drawn from Canadian-based men and the research design was cross-sectional in nature, recruiting a self-selected online population. Longitudinal and geographically representative cross-country data would provide a more comprehensive test of the present mediation effect, as would use of a larger sample to ensure generalisability across the prostate cancer survivor population. Additionally, future work should consider an adjunctive non-online approach to data collection to ensure men without ready internet access (eg, men aged $>70$ years) are also able to participate.

In seeking to better understand the psychosocial experiences of men with prostate cancer, emotion suppression appears a salient factor in the anger-depression relationship. Given men with prostate cancer are at markedly higher risk of depression and suicide attempt, greater emphasis on exploring the utilization of different psychosocial approaches is needed. Taking a gendered lens to the assessment and treatment of mood-related symptoms of depression, including externalised or atypical presentations, may support improved outcomes in this population. The links between anger, emotion suppression and depression in the context of prostate cancer require further attention.

\section{Funding}

This study was supported by the Canadian Institutes of Health Research (Grant 11R06913). Simon Rice is supported by a Career Development Fellowship from the National Health and Medical Research Council of Australia (APP1158881), and the Dame Kate Campbell Fellowship 
from the Faculty of Medicine, Dentistry and Health Sciences at The University of Melbourne.

\section{Disclosure}

The authors report no conflicts of interest in this work.

\section{References}

1. Jayadevappa R, Malkowicz SB, Chhatre S, Johnson JC, Gallo JJ. The burden of depression in prostate cancer. Psychooncology. 2012;21 (12):1338-1345. doi:10.1002/pon.v21.12

2. Nead KT, Sinha S, Yang DD, Nguyen PL. Association of androgen deprivation therapy and depression in the treatment of prostate cancer: a systematic review and meta-analysis. Urol Oncol. 2017;35 (11):664.e661-664.e669. doi:10.1016/j.urolonc.2017.07.016

3. Hartung T, Brähler E, Faller H, et al. The risk of being depressed is significantly higher in cancer patients than in the general population: prevalence and severity of depressive symptoms across major cancer types. Eur J Cancer. 2017;72:46-53. doi:10.1016/j.ejca.2016.11.017

4. Watts S, Leydon G, Birch B, et al. Depression and anxiety in prostate cancer: a systematic review and meta-analysis of prevalence rates. BMJ Open. 2014;4(3):e003901. doi:10.1136/bmjopen-2013-003901

5. Northouse LL, Mood DW, Montie JE, et al. Living with prostate cancer: patients' and spouses' psychosocial status and quality of life. $J$ Clin Oncol. 2007;25(27):4171-4177. doi:10.1200/JCO.2006.09.6503

6. Roth AJ, Weinberger MI, Nelson CJ. Prostate cancer: quality of life, psychosocial implications and treatment choices. Future Oncol. 2008;4(4):561-568. doi:10.2217/14796694.4.4.561

7. Chambers SK, Hyde MK, Smith DP, et al. New challenges in psychooncology research III: a systematic review of psychological interventions for prostate cancer survivors and their partners: clinical and research implications. Psychooncology. 2017;26(7):873-913. doi:10.1002/pon. v26.7

8. Dinh KT, Gally Reznor VM, Mahal BA, et al. Association of androgen deprivation therapy with depression in localized prostate cancer. $J$ Clin Oncol. 2016;34(16):1905-1912. doi:10.1200/JCO.2015.64.1969

9. Sharpley CF, Christie DR, Bitsika V, Miller BJ. Trajectories of total depression and depressive symptoms in prostate cancer patients receiving six months of hormone therapy. Psychooncology. 2017;26 (1):60-66. doi:10.1002/pon.4100

10. Watts S, Leydon G, Eyles C, et al. A quantitative analysis of the prevalence of clinical depression and anxiety in patients with prostate cancer undergoing active surveillance. BMJ Open. 2015;5(5): e006674. doi:10.1136/bmjopen-2014-006674

11. Paterson C, Robertson A, Nabi G. Exploring prostate cancer survivors' self-management behaviours and examining the mechanism effect that links coping and social support to health-related quality of life, anxiety and depression: a prospective longitudinal study. Eur J Oncol Nurs. 2015;19(2):120-128. doi:10.1016/j.ejon.2014.10.008

12. Sharpley CF, Bitsika V, Denham JW. Factors associated with feelings of loss of masculinity in men with prostate cancer in the RADAR trial. Psychooncology. 2014;23(5):524-530. doi:10.1002/pon.v23.5

13. Fervaha G, Izard JP, Tripp DA, Rajan S, Leong DP, Siemens DR. Depression and prostate cancer: a focused review for the clinician. Urol Oncol. 2019;37(4):282-288. doi:10.1016/j.urolonc.2018.12.020

14. Satin JR, Linden W, Phillips MJ. Depression as a predictor of disease progression and mortality in cancer patients: a meta-analysis. Cancer. 2009;115(22):5349-5361. doi:10.1002/cncr.24561

15. Klaassen Z, Arora K, Wilson SN, et al. Decreasing suicide risk among patients with prostate cancer: implications for depression, erectile dysfunction, and suicidal ideation screening. Urol Oncol. 2018;36(2):60-66. doi:10.1016/j.urolonc.2017.09.007
16. American Psychiatric Association. Diagnostic and Statistical Manual of Mental Disorders $\left(D S M-5^{\mathbb{R}}\right)$. Arlington, VA: American Psychiatric Pub; 2013.

17. World Health Organization. The ICD-10 Classification of Mental and Behavioural Disorders: Clinical Descriptions and Diagnostic Guidelines. Geneva: World Health Organization; 1992.

18. Oliffe JL, Rossnagel E, Seidler ZE, Kealy D, Ogrodniczuk JS, Rice SM. Men's depression and suicide. Curr Psychiatry Rep. 2019;21(10):103. doi:10.1007/s11920-019-1088-y

19. Rutz W, Rihmer Z. Education of general practitioners in depression and suicide prevention. In: Wasserman D, editor. "Suicide: An Unnecessary Death. Vol. 2. Oxford University Press; 2016:309.

20. Martin LA, Neighbors HW, Griffith DM. The experience of symptoms of depression in men vs women: analysis of the National Comorbidity Survey Replication. JAMA Psychiatry. 2013;70 (10):1100-1106. doi:10.1001/jamapsychiatry.2013.1985

21. Cavanagh A, Wilson CJ, Kavanagh DJ, Caputi P. Differences in the expression of symptoms in men versus women with depression: a systematic review and meta-analysis. Harv Rev Psychiatry. 2017;25(1):29-38. doi:10.1097/HRP.0000000000000128

22. Genuchi M. Anger and hostility as primary externalizing features of depression in college men. Int J Men's Health. 2015;14(2):113-128.

23. Heifner C. The male experience of depression. Perspect Psychiatr Care. 1997;33(2):10-18. doi:10.1111/j.1744-6163.1997.tb00536.x

24. Brownhill S, Wilhelm K, Barclay L, Schmied V. 'Big build': hidden depression in men. Aust N Z J Psychiatry. 2005;39(10):921-931. doi:10.1080/j.1440-1614.2005.01665.x

25. Rice S. Depression in Men: Development of the Male Depression Risk Scale. Australia: School of Psychology, Australian Catholic University; 2011.

26. Magovcevic M, Addis ME. The masculine depression scale: development and psychometric evaluation. Psychol Men Masc. 2008;9 (3):117-132. doi:10.1037/1524-9220.9.3.117

27. Rice SM, Fallon BJ, Aucote HM, Möller-Leimkühler A, Treeby MS, Amminger GP. Longitudinal sex differences of externalising and internalising depression symptom trajectories: implications for assessment of depression in men from an online study. Int J Soc Psychiatry. 2015;61 (3):236-240. doi:10.1177/0020764014540149

28. Kuehner C. Is it all depression? JAMA Psychiatry. 2014;71(3):337. doi:10.1001/jamapsychiatry.2013.4334

29. Gerhart J, Schmidt E, Lillis T, O’Mahony S, Duberstein P, Hoerger M. Anger proneness and prognostic pessimism in men with prostate cancer. Am J Hosp Palliat Care. 2017;34(6):497-504. doi:10.1177/10499 09116636358

30. Pene $\mathrm{CTH}$, Kissane D. Communication in cancer: its impact on the experience of cancer care: communicating with the angry patient and the patient in denial. Curr Opin Support Palliat Care. 2019;13(1):46-52. doi:10.1097/SPC.0000000000000410

31. Morris T, Greer S, Pettingale KW, Watson M. Patterns of expression of anger and their psychological correlates in women with breast cancer. J Psychosom Res. 1981;25(2):111-117. doi:10.1016/00223999(81)90098-2

32. Weber KM, Solomon DH. Locating relationship and communication issues among stressors associated with breast cancer. Health Commun. 2008;23(6):548-559. doi:10.1080/10410230802465233

33. Lieberman MA, Goldstein BA. Not all negative emotions are equal: the role of emotional expression in online support groups for women with breast cancer. Psychooncology. 2006;15(2):160-168. doi:10.1002/(ISSN) 1099-1611

34. McKenna MC, Zevon MA, Corn B, Rounds J. Psychosocial factors and the development of breast cancer: a meta-analysis. Health Psychol. 1999;18(5):520-531. doi:10.1037/0278-6133.18. 5.520

35. Groarke A, Curtis R, Walsh DM, Sullivan FJ. What predicts emotional response in men awaiting prostate biopsy? BMC Urol. 2018;18 (1):27. doi:10.1186/s12894-018-0340-9 
36. Gross J. Emotional expression in cancer onset and progression. Soc Sci Med. 1989;28(12):1239-1248. doi:10.1016/0277-9536(89)90342-0

37. Penedo FJ, Dahn JR, Kinsinger D, et al. Anger suppression mediates the relationship between optimism and natural killer cell cytotoxicity in men treated for localized prostate cancer. J Psychosom Res. 2006;60(4):423-427. doi:10.1016/j.jpsychores.2005.08.001

38. Thomas SP, Groer M, Davis M, Droppleman P, Mozingo J, Pierce M. Anger and cancer: an analysis of the linkages. Cancer Nurs. 2000;23 (5):344-349. doi:10.1097/00002820-200010000-00003

39. White VM, English DR, Coates H, Lagerlund M, Borland R, GG G. Is cancer risk associated with anger control and negative affect? Findings from a prospective cohort study. Psychosom Med. 2007;69 (7):667-674. doi:10.1097/PSY.0b013e31814d4e6a

40. Kreitler S, editor. Psychosocial risk factors for cancer. In: PsychoOncology for the Clinician. Springer; 2019:63-84.

41. Sigmund F, James S. Civilization and its discontents. Stand Ed. 1930;21:64-145.

42. Kassinove H, Sukhodolsky DG. Anger disorders: basic science and practice issues. Issues Compr Pediatr Nurs. 1995;18(3):173-205. doi:10.3109/01460869509087270

43. Cassiello-Robbins C, Barlow DH. Anger: the unrecognized emotion in emotional disorders. Clin Psychol. 2016;23(1):66-85.

44. Hayes AF. Introduction to Mediation, Moderation, and Conditional Process Analysis: A Regression-Based Approach. Second ed. New York, NY: Guilford Publications; 2017.

45. Rice SM, Fallon BJ, Aucote HM, Möller-Leimkühler AM. Development and preliminary validation of the male depression risk scale: furthering the assessment of depression in men. $J$ Affect Disord. 2013;151(3):950-958. doi:10.1016/j.jad.2013.08.013

46. Rice SM, Ogrodniczuk JS, Kealy D, Seidler ZE, Dhillon HM, Oliffe JL. Validity of the male depression risk scale in a representative Canadian sample: sensitivity and specificity in identifying men with recent suicide attempt. J Ment Health. 2019;28(2):132-140. doi:10.1080/09638 237.2017 .1417565
47. Rice SM, Oliffe JL, Kelly MT, et al. Depression and prostate cancer: examining comorbidity and male-specific symptoms. Am J Men's Health. 2018;12(6):1864-1872. doi:10.1177/1557988318784395

48. Kroenke K, Spitzer RL, Williams JB. The PHQ-9: validity of a brief depression severity measure. J Gen Intern Med. 2001;16(9):606-613. doi:10.1046/j.1525-1497.2001.016009606.x

49. Kocalevent R-D, Hinz A, Brähler E. Standardization of the depression screener Patient Health Questionnaire (PHQ-9) in the general population. Gen Hosp Psychiatry. 2013;35(5):551-555. doi:10.1016/ j.genhosppsych.2013.04.006

50. Cohen J. Statistical Power Analysis for the Behavioral Sciences. Second ed. Abingdon, UK: Routledge; 2013. [1999].

51. Movember. Perceptions of Masculinity \& the Challenges of Opening Up: Global Research Report. Melbourne: Movember; 2019.

52. Wall D, Kristjanson L. Men, culture and hegemonic masculinity: understanding the experience of prostate cancer. Nurs Inq. 2005;12 (2):87-97. doi:10.1111/nin.2005.12.issue-2

53. Seidler ZE, Lawsin CR, Hoyt MA, Dobinson KA. Let's talk about sex after cancer: exploring barriers and facilitators to sexual communication in male cancer survivors. Psycho-oncology. 2016;25 (6):670-676. doi:10.1002/pon.v25.6

54. Chambers SK, Foley E, Galt E, Ferguson M, Clutton S. Mindfulness groups for men with advanced prostate cancer: a pilot study to assess feasibility and effectiveness and the role of peer support. Support Care Cancer. 2012;20(6):1183-1192. doi:10.1007/s00520-011-1195-8

55. Berke DS, Zeichner A. Man's heaviest burden: a review of contemporary paradigms and new directions for understanding and preventing masculine aggression. Soc Personal Psychol Compass. 2016;10 (2):83-91. doi:10.1111/spc3.v10.2

56. Oliffe JL, Rossnagel E, Bottorff JL, Chambers SK, Caperchione C, Rice SM. Community-based men's health promotion programs: eight lessons learnt and their caveats. Health Promot Int. 2019. doi:10.10 93/heapro/daz101

\section{Publish your work in this journal}

Cancer Management and Research is an international, peer-reviewed open access journal focusing on cancer research and the optimal use of preventative and integrated treatment interventions to achieve improved outcomes, enhanced survival and quality of life for the cancer patient.
The manuscript management system is completely online and includes a very quick and fair peer-review system, which is all easy to use. Visit http://www.dovepress.com/testimonials.php to read real quotes from published authors. 